\title{
The Impact of Patient Age on The Acceptability of Telemedicine in The Context Of COVID-19
}

\author{
Briana Williams, Katelin McDilda, \& Melissa Bright \\ College of Medicine
}

Faculty Mentor: Reem S. Abu-Rustum, Department of Obstetrics \& Gynecology

\begin{abstract}
The objective of the study was to determine the extent to which patients from various age groups perceive telemedicine as a viable mode of healthcare delivery in the context of COVID-19. A RedCap survey was sent to patients in our OB/GYN outpatient clinics with in-person, telemedicine, re-scheduled or cancelled appointments between 3/11/20 to 5/11/20. Patients' online responses were analyzed using a 5-point Likert scale. Statistical analysis was performed using Chi Square and Fischer's Exact Analysis with $\mathrm{p}<0.05$ considered significant. A total of 1083 patients completed the survey of whom 280 (25.9\%) had a telemedicine appointment. Patients answered questions relating to their telemedicine visit. While older patients did encounter a higher proportion of technological difficulties $(\mathrm{p}<0.0001)$, younger patients, specifically those in the 25-34 age group, expressed greater dissatisfaction with their appointment being changed to telemedicine than older patients $(\mathrm{p}=0.02)$, and felt that telemedicine did not accomplish the same goals as an in-person visit $(\mathrm{p}=0.01)$. Nonetheless, all patients, regardless of age, were satisfied with the introduction to telemedicine $(\mathrm{p}=0.02)$ and the instructions provided to them prior to the visit $(\mathrm{p}=0.02)$. Connectivity issues seem to be the biggest obstacle to older patients particularly when there is absence of a reliable internet connection and a telemedicine-compatible device. Younger patients, though less satisfied, are more comfortable with new technology and with using telemedicine; they tend to experience less connectivity issues. The focus going forward should be on finding ways to simplify the process, overcome the connectivity issues while addressing the main reasons leading to patient dissatisfaction.

Keywords: telemedicine, COVID-19, patient age
\end{abstract}

\section{Introduction}

The COVID-19 public health crisis has triggered a response in healthcare that prioritizes implementing containment and safety measures for patients. Providers have been tasked with finding ways to quickly provide high-quality care for patients while considering the public health crisis. Telemedicine has become one of the most practical courses of action given it minimizes the risks of exposure for both patient and provider by granting providers the ability to remotely deliver care to patients. This is a safer alternative to in-person patient visits (Portnoy et al., 
2020). While telemedicine is an effective means to deliver care, it is rapidly being implemented without accounting for how the different age groups will acclimate to this new technology-based service and the restrictions in the health care setting (no visitor policy).

As such, we hypothesized that given telemedicine relies so heavily on modern technology, adults $65+$ may have a more difficult time adapting to its use. Older individuals tend to have a lesser understanding of technology-based platforms and may be less accepting of new innovations (Cimperman et al., 2013). Furthermore, older adults may be further hindered in their use of telemedicine due to age-related changes in their vision, hearing, and dexterity (Foster \& Sethares, 2014). Therefore, the new adoption of telemedicine poses a challenge for this population. Conversely, young adults are more accepting and welcoming of the convenience of telemedicine due to their proficiency with electronic and online communication platforms (Barney et al., 2020).

Additionally, many existing telemedicine services were designed and approved without considering patient perspectives on functionality and utility (Foster \& Sethares, 2014). With this, there is a potential for telemedicine to affect the quality and access to care. Consequently, there is a clear need for research in this area to determine how patients are handling the implementation of telemedicine. This study will assess how patients from various age groups perceive the ease of use and quality of care delivered through telemedicine.

\section{Study Design}

An online RedCap survey was distributed to patients with an appointment scheduled at an academic center's OB/GYN outpatient clinics. The appointment was scheduled for in-person, telemedicine, re-scheduled or cancelled between $3 / 11 / 20$ to 5/11/20, during the height of the initial COVID-19 outbreak in Florida. Questions addressed demographics, reason for appointment, COVID-19 precautions and exposures, encounters with new COVID-19 policies and practices within the clinic, and satisfaction and concerns with these policies and practices. All questions had a "choose not to answer" as an option. This led to varying sample sizes by question. Patients' online responses were recorded using a 5-point Likert scale, or a yes-no question format. Statistical analysis was determined using Chi Square and Fischer's Exact Analysis with $\mathrm{p}<0.05$ considered significant. Furthermore, the reliability and validity were not 
calculated because the total or composite measure of perceptions for telemedicine were not created.

\section{Results}

Of the 1083 patients that completed the survey, 280 (25.9\%) responded "yes" to having a telemedicine appointment and completed the questions pertaining to telemedicine use. Of the 280 telehealth respondents, 155 were pregnant, as shown in Table 1. Respondents that were pregnant were as follows: $10.3 \%$ of the $15-24$ age group, $67.7 \%$ of the $25-34$ age group, and $21.9 \%$ of the $35-44$ age group.

Table 1. Patient Demographics

\begin{tabular}{ccc}
\hline $\begin{array}{c}\text { Patient Age } \\
\text { Group (years) }\end{array}$ & $\begin{array}{c}\text { Total Telehealth } \\
\text { Respondents }\end{array}$ & $\begin{array}{c}\text { Total } \\
\text { Pregnant }\end{array}$ \\
\hline $15-24$ & $27(9.6 \%)$ & $16(10.3 \%)$ \\
$25-34$ & $117(41.8 \%)$ & $105(67.7 \%)$ \\
$35-44$ & $66(23.6 \%)$ & $34(21.9 \%)$ \\
$45-54$ & $34(12.1 \%)$ & 0 \\
$55-64$ & $19(6.8 \%)$ & 0 \\
$65+$ & $17(6.1 \%)$ & 0 \\
\hline Total & $280(100 \%)$ & $155(100 \%)$ \\
\hline
\end{tabular}

When asked if relieved by the telemedicine appointment, $58.8 \%$ of patients in the $65+$ age group reported they were relieved by their in-person visit being converted to telemedicine versus a smaller percentage of the younger age groups (31.6\% - 53\%). Ease of telemedicine use is related to patient age, with younger patients reporting fewer issues utilizing telemedicine. Despite fewer issues related to utilization, younger patients expressed dissatisfaction with having 
a telemedicine instead of an in-person visit as follows: $30.8 \%$ in the $25-34$ age group in comparison to $17.6 \%$ in the $65+$ age group (Table 2 and Figure 1).

Table 2. Dissatisfaction with Telemedicine by Age Group

\begin{tabular}{ccccccc}
\hline Age Group & Strongly Disagree & Disagree & Neutral & Agree & Strongly Agree & Total \\
\hline $15-24$ & $10(3.57 \%)$ & $4(1.43 \%)$ & $7(2.5 \%)$ & $6(2.14 \%)$ & $0(0 \%)$ & 27 \\
$25-34$ & $25(8.93 \%)$ & $32(11.43 \%)$ & $24(8.57 \%)$ & $19(6.79 \%)$ & $17(6.07 \%)$ & 117 \\
$35-44$ & $19(6.79 \%)$ & $24(8.57 \%)$ & $14(15 \%)$ & $2(0.71 \%)$ & $7(2.5 \%)$ & 66 \\
$45-54$ & $14(5 \%)$ & $13(4.64 \%)$ & $5(1.79 \%)$ & $0(0 \%)$ & $2(0.71 \%)$ & 34 \\
$55-64$ & $8(2.86 \%)$ & $6(2.14 \%)$ & $3(1.07 \%)$ & $1(0.36 \%)$ & $1(0.36 \%)$ & 19 \\
$65+$ & $8(2.86 \%)$ & $5(1.79 \%)$ & $1(0.36 \%)$ & $2(0.71 \%)$ & $1(0.36 \%)$ & 17 \\
\hline Total & 84 & 84 & 54 & 30 & 28 & 280 \\
\hline
\end{tabular}

Figure 1. Dissatisfaction with Telemedicine by Age Group

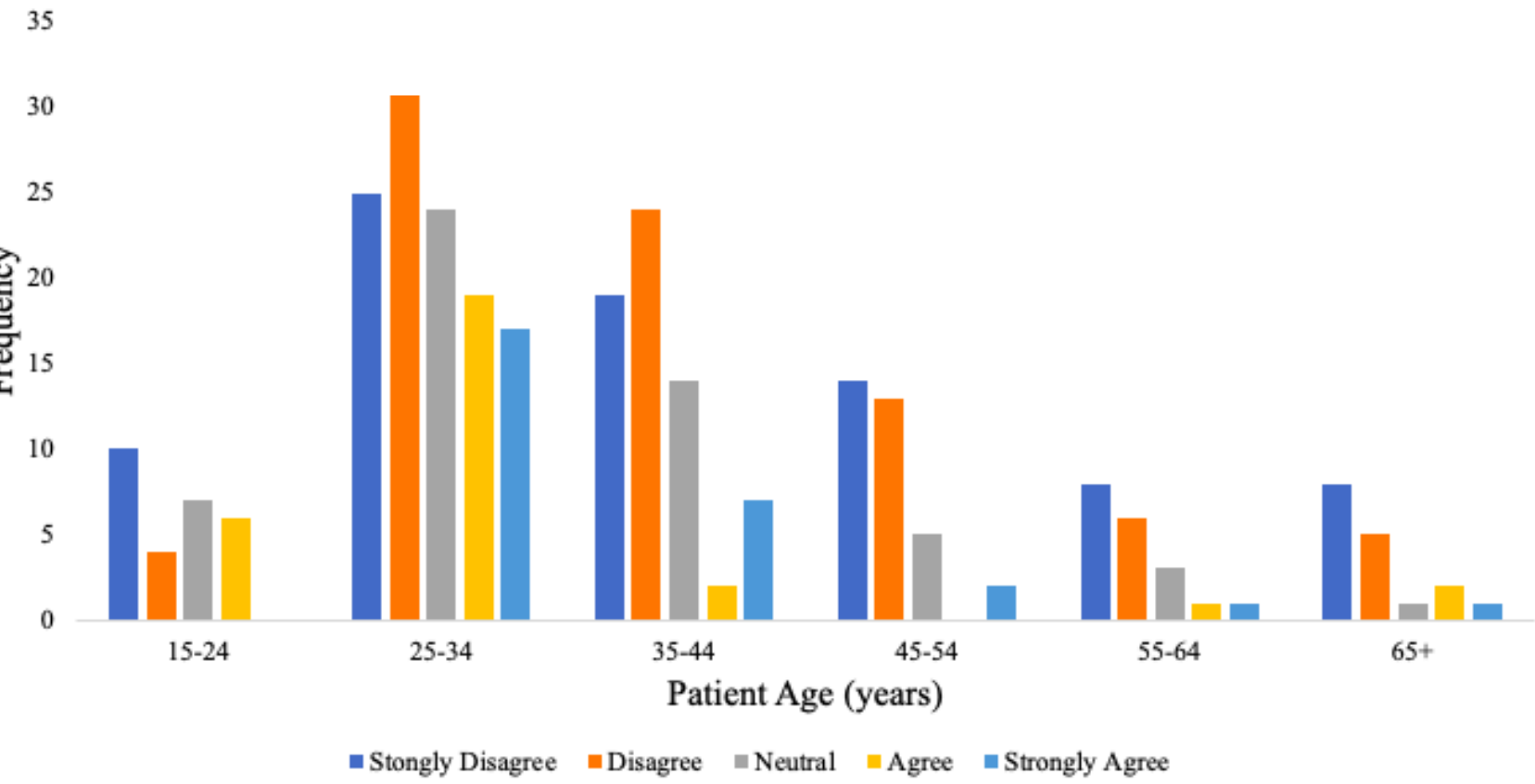


Younger patients were satisfied with the introductions given for telemedicine visits $(p=0.02)$ and felt the instructions for connecting to the appointment were clear $(p=0.02)$. In addition, younger patients felt it was easy to connect to their telemedicine appointments $(p=0.009)$ and encountered less difficulties doing so ( $<$ 0.0001) (Table 3 and Figure 2). Of the difficulties encountered, the most frequent was a technological concern with audio and/or video in $6.9 \%$ on the patient side versus $5 \%$ on the provider side.

Table 3. Connection Difficulties to Telemedicine by Patient Age

\begin{tabular}{cccc}
\hline Patient Age & No & Yes & Total \\
\hline $15-24$ & $23(92 \%)$ & $2(8 \%)$ & 25 \\
$25-34$ & $93(82.3 \%)$ & $20(17.7 \%)$ & 113 \\
$35-44$ & $58(92.1 \%)$ & $5(7.9 \%)$ & 63 \\
$45-54$ & $24(92.3 \%)$ & $2(7.7 \%)$ & 26 \\
$55-64$ & $16(88.9 \%)$ & $2(11.1 \%)$ & 18 \\
$65+$ & $7(43.8 \%)$ & $9(56.2 \%)$ & 16 \\
\hline Total & 221 & 40 & 261 \\
\hline
\end{tabular}


Figure 2. Connection Difficulties by Age Group

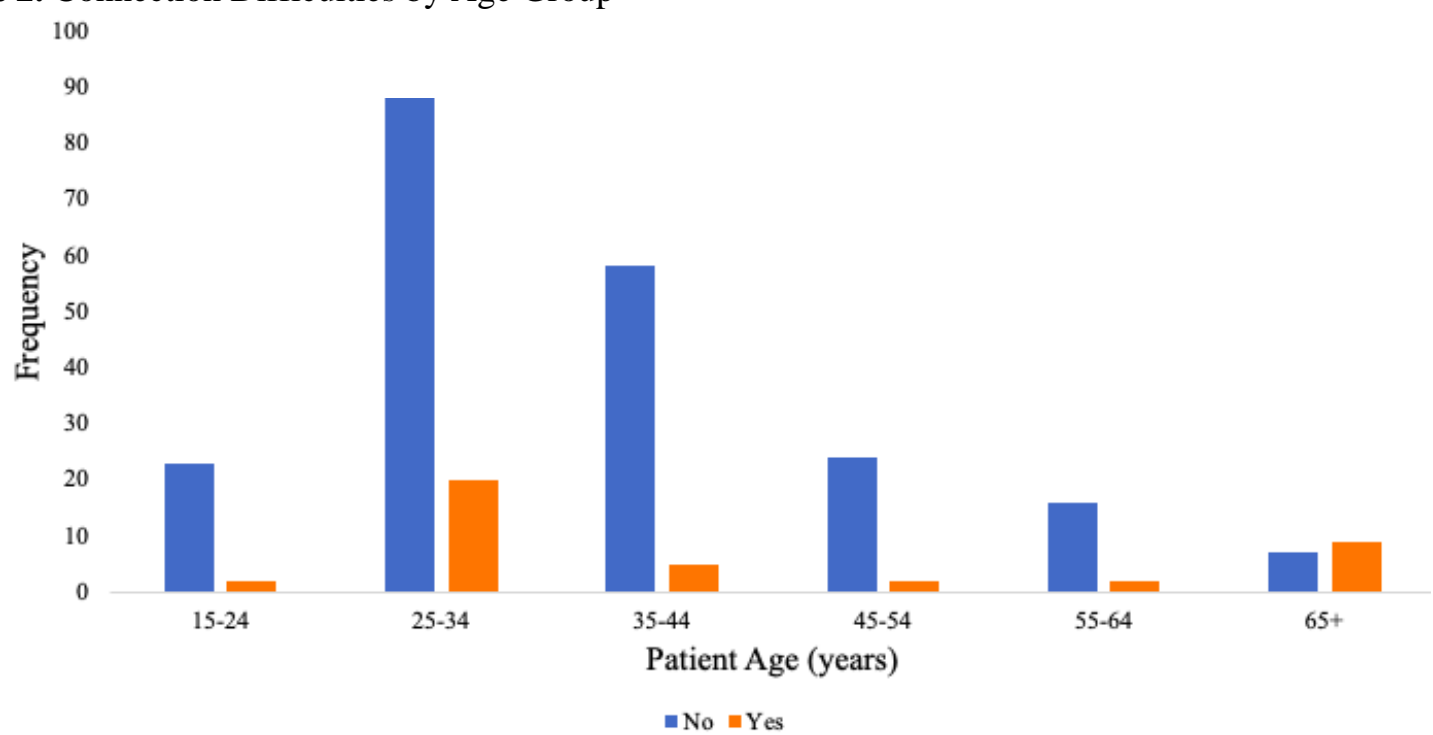

Even though telemedicine was more easily accessed by younger patients, younger patients disagreed that telemedicine accomplished the same goals as an in-person visit (Figure 3) where $63.2 \%$ of those in the $25-34$ age group versus $29-47 \%$ in the older age groups disagreed that telemedicine accomplished the same goals as an in-person visit.

Figure 3. Patient Goals Met by Telemedicine

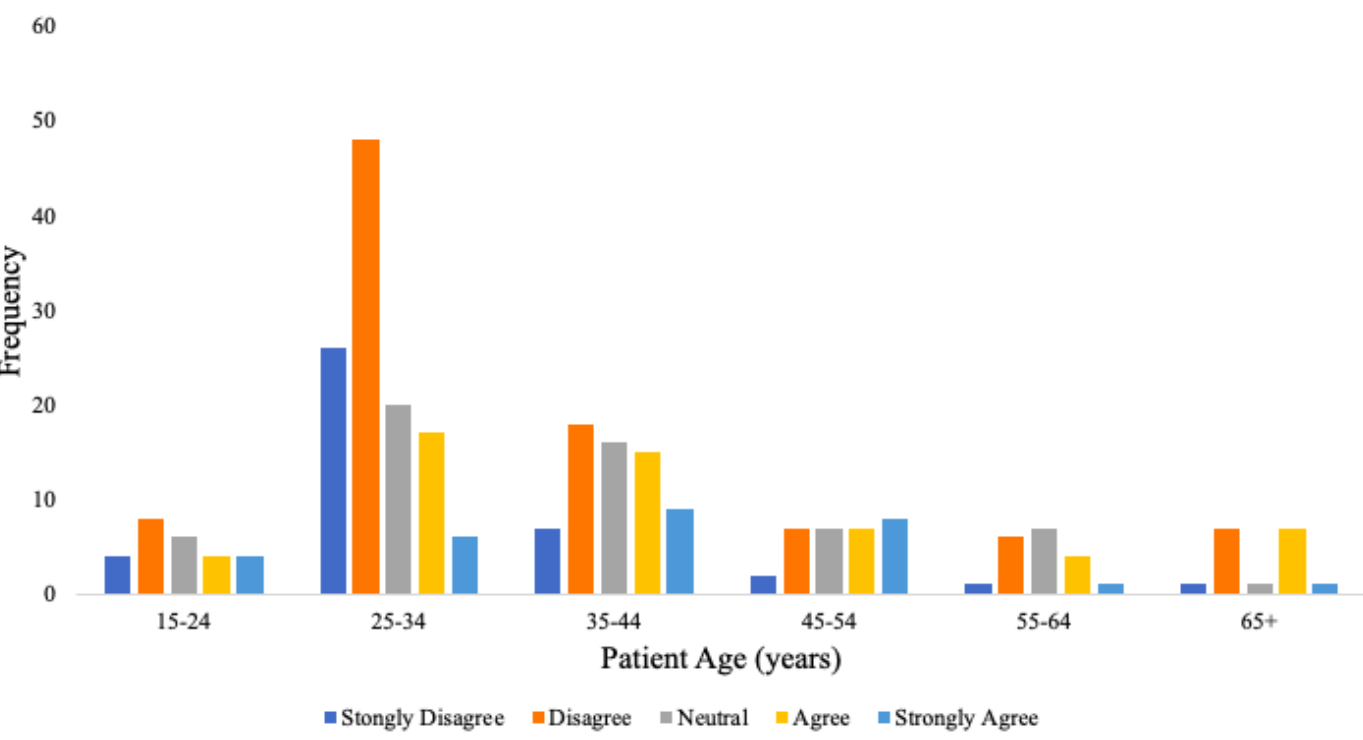




\section{Discussion}

Telemedicine was greatly utilized during the COVID-19 pandemic creating a large pool of patient experiences to evaluate. Our results show that younger patients, particularly the 25-34 age group were the most dissatisfied by having their in-person appointment converted to a telemedicine appointment. This may be explained by the fact that younger patients tend to have prenatal or pregnancy-related issues as the reason for their visit. A telemedicine visit would not enable measuring fundal height to ensure proper fetal growth, nor would it allow hearing the fetal heartbeat or seeing the fetus sonographically. Increased reporting of being dissatisfied with the telemedicine appointment by the 25-34 age group could also be associated with the actual and perceived lower personal risk COVID-19 posed to this age group.

Overall, the younger population is more likely to be proficient in technological-based platforms. The number of patients below the age of 65 that found it easy to connect to their telemedicine appointment outweighed the number of patients that had difficulties connecting. While in the $65+$ age group, a larger proportion of patients found it difficult to connect to their telemedicine appointment compared to those that did not have connection difficulties. Furthermore, the 65+ age group is the only group that reported having more difficulties connecting to their appointment. Generally, patients of all ages agreed that the instructions to use telemedicine were clear. Therefore, the issues experienced by the older age group may not have been addressed by the survey as they may have been attributed to age-related auditory, visual, or dexterity limitations. The most common difficulty encountered amongst all age groups was a connectivity problem on the patient's side making a solution challenging since telemedicine relies on many socioeconomic resources that cannot be easily accounted for.

Furthermore, there were a few limitations within the study including a low patient response rate particularly for reproductive and endocrinology patients who had cancelled appointments during the period of time when the survey was sent out. The surveys were also only sent to patients with an email address on file. Therefore, there is little information on patients who were not sent the survey or who did not fill out the survey. It is also important to consider inherent selection bias within the study because it is possible that patients were more likely to complete the survey if they were more comfortable with using technology or had fewer connectivity issues. 
Overall, OB/GYN patients with telemedicine appointments were satisfied with their experience with telemedicine but felt that telemedicine appointments could not accomplish the same goals as an in-person visit. This was consistent across all age groups. Patients often associate OB/GYN visits with physical exams including pelvic exams, fundal height, doptones and songraphic evaluations. The lack of physical exam available through telemedicine may not meet patient expectations when scheduling an $\mathrm{OB} / \mathrm{GYN}$ appointment. Many pregnant patients look forward to having family members present during prenatal and ultrasound appointments. Subsequently, the inability to accommodate this may have led to overall dissatisfaction with telemedicine.

\section{Conclusion}

The COVID-19 pandemic has resulted in increased use of telemedicine as a viable option to deliver care. However, telemedicine poses various challenging issues for different age groups with respect to connectivity and satisfaction with the care provided. Predominantly, younger patients were more dissatisfied with having a telemedicine appointment as opposed to an inperson appointment and felt their goals were not met through telemedicine. Patients also need to have a reliable internet connection and a telemedicine-compatible device. In general, younger patients are more comfortable with telemedicine and experience less connectivity issues than older patients. Therefore, the future focus should be on finding ways to simplify the process, address the connectivity issues and find means for remote reassurance of fetal well-being. Further research is needed to assess patient preferences for optimal equitable remote care, particularly for younger and pregnant patients.

\section{Acknowledgements}

We wish to acknowledge John C. Smulian, MD, MPH, Nash Moawad, MD, Emily Webur-LeBrun, MD, Kay Roussos-Ross, MD, Gregory Christman, MD, and Alice Rhoton-Vlasak, MD for their contributions to this study.

\section{References}

Barney, A., Buckelew, S., Mesheriakova, V., \& Raymond-Flesch, M. (2020). The COVID-19 pandemic and rapid implementation of adolescent and young adult telemedicine: Challenges and opportunities for innovation. The Journal of adolescent health: official publication of the Society for Adolescent Medicine, 67(2), 164-171. https://doi.org/10.1016/j.jadohealth.2020.05.006 
Cimperman, M., Brenčič, M. M., Trkman, P., \& Stanonik, M. (2013). Older adults' perceptions of home telehealth services. Telemedicine journal and e-health: the official journal of the American Telemedicine Association, 19(10), 786-790. https://doi.org/10.1089/tmj.2012.0272

Foster, M. V., \& Sethares, K. A. (2014). Facilitators and barriers to the adoption of telehealth in older adults: an integrative review. Computers, informatics, nursing: CIN, 32(11), 523-535. https://doi.org/10.1097/CIN.0000000000000105

Portnoy, J., Waller, M., \& Elliott, T. (2020). Telemedicine in the era of COVID-19. The journal of allergy and clinical immunology. In practice, 8(5), 1489-1491. https://doi.org/10.1016/j.jaip.2020.03.008 